rupture is usually a myxomatous mitral valve in which the mechanical properties of the chordae are affected.

There are several indications of a genetic link to myxomatous mitral valve disease. Thus patients with autosomal dominant polycystic kidney disease have an increased occurrence of cardiac valve abnormalities, and in patients with Marfan syndrome caused by fibrillin gene defects, myxomatous mitral valve disease is the leading cause of mitral regurgitation. Moreover, a high frequency of the angiotensin-converting enzyme II genotype has been demonstrated in myxomatous mitral valve disease, and a gene associated with the disease has been located on Xq28. ${ }^{3}$

The presented patients had remarkably similar morphologic findings, with a large myxomatous valve and posterior leaflet prolapse combined with chordal rupture. Both of the patients, in addition, had an enlargement of the left ventricle. Eliminating the $\mathrm{MR}$, if performed early, will reduce the volume overload, allowing the left ventricle to recover in size and function. The timing of surgical intervention in asymptomatic patients might be difficult, but when the EF decreases to $60 \%$ or less, the LVESD increases to greater than $40 \mathrm{~mm}$, or both, surgical intervention is advocated. ${ }^{4}$

Patient 2 came to surgical intervention almost a year later than patient 1 and was found to have a more severely enlarged heart. In view of the importance of promoting ventricular reversed remodeling and ensuring a reduction of ventricular size to minimize the risk of potential residual functional MR, the CSD was positioned around the heart. Obviously this proved to be most efficient because the heart rapidly decreased in size with maintained LV function. To what extent passive containment operations should be applied more generally in patients with cardiac LV dilatation remains to be established.

\section{References}

1. Konertz WF, Shapland JE, Hotz H, Dusche S, Braun JP, Stantke K, et al. Passive containment and reverse remodelling by a novel textile cardiac support device. Circulation. 2001;104(suppl):I270-5.

2. Hickey AJ, Wicken DEL, Wright JS, Warren BA. Primary (spontaneous) chordal rupture: relation to myxomatous valve disease and mitral valve prolapse. J Am Coll Cardiol. 1985;5:1341-6.

3. Mieno S, Horimoto H, Asada K, Sasaki S. Simultaneous onset of mitral valve regurgitation requiring surgery due to primary chordal rupture in middle aged identical twins. Int J Cardiol. 2005;103:214-6.

4. Carabello BA. Indications for mitral valve surgery. J Cardiovasc Surg. 2004;45:407-18.

\title{
A fibrous membrane causing left ventricular outflow tract stenosis as the result of endocarditis
}

\author{
Anita Pritisanac, MD, Andreas Hannekum, MD, and Helmut Gulbins, MD
}

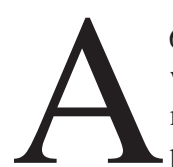

68-year-old man with a history of open heart surgery was admitted to our cardiothoracic surgery department because of fever, dyspnea, and reduced cardiac performance during the last 2 months. Six years ago, the patient underwent biologic aortic valve replacement because of aortic insufficiency, conduit implantation because of aortic aneurysm, and aortocoronary bypasses in our department.

\section{Case Report}

The patient had been admitted to the hospital 4 weeks before with suspected bacteremia. Four teeth were suspected as the focus of infection and were extracted. During that period he showed signs of

From the Department of Cardiothoracic Surgery, University of Ulm, Germany.

Received for publication Nov 3, 2005; accepted for publication Nov 16, 2005.

Address for reprints: Anita Pritisanac, MD, Department of Cardiothoracic Surgery, University Hospital Ulm, Steinhövelstr 9, 89075 Ulm, Germany (E-mail: anita.pritisanac@medizin.uni-ulm.de).

J Thorac Cardiovasc Surg 2006;131:1401-2

$0022-5223 / \$ 32.00$

Copyright $\odot 2006$ by The American Association for Thoracic Surgery doi:10.1016/j.jtcvs.2005.11.057 infection. Along with elevated temperatures blood cultures were drawn and revealed Streptococcus oralis. Transthoracic echocardiography showed no pathologic findings. Cardiac auscultation was repeatedly without pathologic murmurs. Electrocardiography showed no new pathologic findings and no signs of myocardial ischemia. The maximum temperature was $37.9^{\circ} \mathrm{C}$. Laboratory investigations showed leukocytosis (17.000 Giga/L). C-reactive protein was $27 \mathrm{mg} / \mathrm{L}$ at maximum and urine analysis turned out to be normal. The chest x-ray showed no pathology. Repeated transesophageal echocardiography showed no vegetations or abscess of the biologic aortic prosthesis, and no paravalvular leakage. The mitral valve was also normal. A slightly impaired motion of all 3 cusps and a peak-to-peak gradient of $60 \mathrm{~mm} \mathrm{Hg}$ were findings of aortic valve stenosis, which had not been documented before. Ejection fraction was $50 \%$, as in the former study. Heart catheterization showed no pathologic findings. Because the clinical condition of the patient worsened without response to medical treatment, a surgical intervention was scheduled.

The suspected leading preoperative pathologic condition was an impaired cusp motility of the aortic prosthesis caused by acute endocarditis of the valve or aortic conduit, probably explaining the new findings of aortic valve stenosis.

In contrast, the intraoperative appearance of the conduit and aortic valve showed no signs of infection. The prosthesis was free of vegetations or abscess; the leaflet anatomy and its function were 


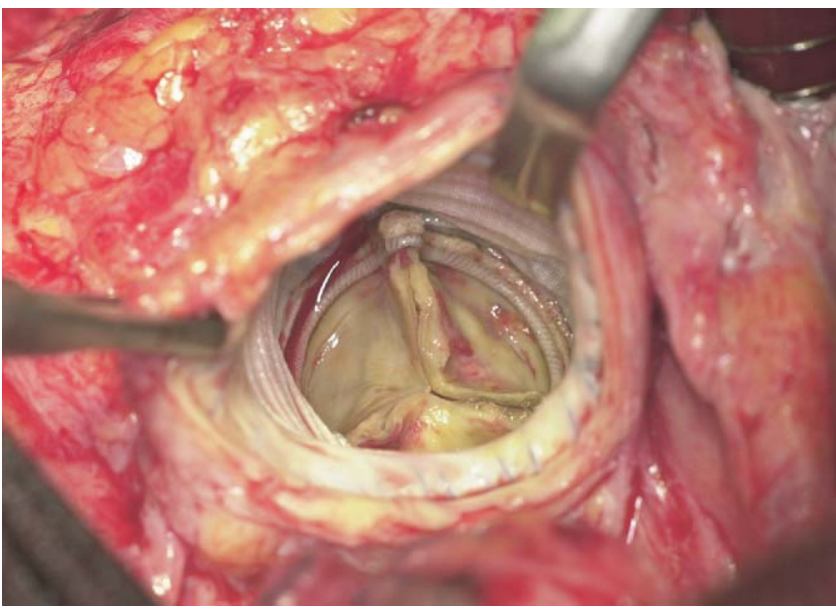

Figure 1. Bioprosthesis showed no macroscopic signs of endocarditis with normal leaflet anatomy and no vegetations or abscess.

unremarkable (Figure 1). A rigid circular fibrous membrane was found below the annulus of the aortic prosthesis with no signs of infection. This fibrous membrane seemed to have impaired the aortic valve cusp movement and caused the pressure gradient between the left ventricle and the aorta (Figure 2). The membrane was excised completely and a specimen was sent for histologic and microbiologic investigation. The patient received a new conduit and mechanical prosthesis. Postoperative transesophageal echocardiography showed normal function of the new mechanical valve with no signs of stenosis.

\section{Pathologic Discussion}

Gram stain showed gram-positive Staphylococcus oralis on the biologic prosthesis. Specimens of the conduit were sterile, and all parts of the membrane were sterile. Microscopic evaluation showed a fibrous tissue, as it is found in granulation tissue. The valve cusps were infiltrated with fibroblasts and showed widely infiltrated granulocytic areas. It is believed that the membrane led to signs of aortic stenosis. As a result of inflammation, fibrous tissue developed due to acute endocarditis, probably during the time that teeth were extracted. The histopathologic findings estimated the fibrotic tissue to be approximately 2 months old.

\section{Discussion}

The clinical importance of this presented case consists in the discrepancy of the preoperative condition of the patient and intraoperative pathology of a fibrous membrane causing stenosis of the left ventricular outflow tract below the aortic prosthesis. Preoperative echocardiography did not show any vegetations or abscess as clear signs of endocarditis. The examination of the subvalvular region was disturbed by artefacts caused by the prosthetic aortic valve. However, Doppler sonography showed significant aortic stenosis. These findings were confirmed intraoperatively. The sub-

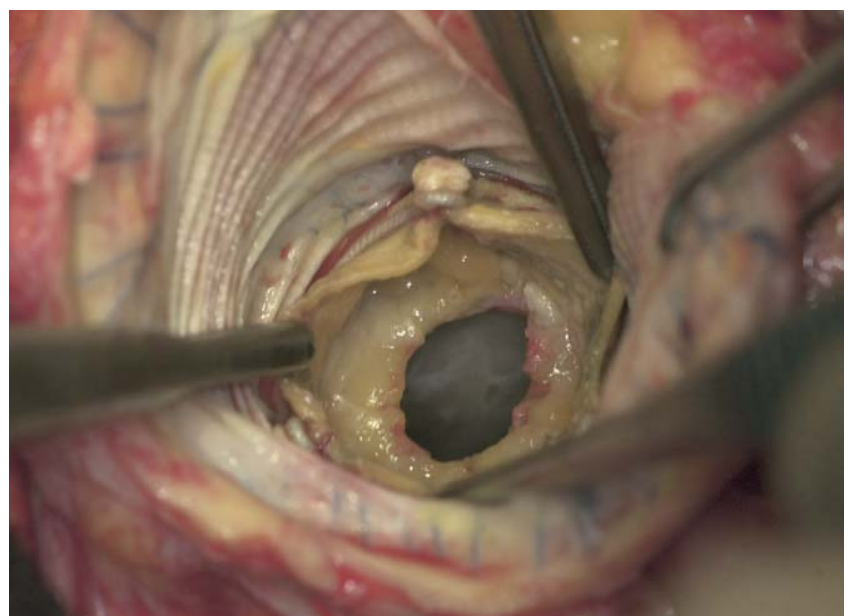

Figure 2. Intraoperative view of the newly developed rigid circular fibrous membrane below the anulus of aortic valve prosthesis.

valvular stenosis could have been caused by 2 mechanisms: Pannus formation resulting from an acute infection or subvalvular fibrosis. The latter is a rare condition, described only in single case reports in pediatric patients. In addition, the fact that no subvalvular stenosis was determined in the echocardiographic investigation 4 weeks before, reveals this pathomechanism to be unlikely. The patient's endocarditis some weeks previously led to the development of this subvalvular stenosis by the mechanism of pannus formation after aortic valve replacement. This case shows an unusual pathoanatomic sequela of an acute endocarditis after prosthetic heart valve replacement and once more underlines the variety of possible presentations of this disease. The patient received 36 days of intravenous antibiotics and recovered with no major complications from surgery. The function of the mechanical valve was excellent, heart function was stable, and blood cultures were sterile. $^{1-5}$

\section{References}

1. Andres E, Baudoux C, Noel E, Goichot B, Schlienger JL, Blickle JF The value of the Von Reyn and the Duke diagnostic criteria for infective endocarditis in internal medicine practice. A study of 38 cases. Eur J Intern Med. 2003;14:411-4.

2. Bayer A, Bolger A, Taubert K, Wilson W, Steckelberg J. Diagnosis and management of infective endocarditis and its complications. Circulation. 1998;98:2936-48.

3. Gulbins H, Kilian E, Roth S, Uhlig A, Kreuzer E, Reichart B. Is there an advantage in using homografts in patients with acute infective endocarditis of the aortic valve? J Heart Valve Dis. 2002;11:492-7.

4. Shapira N, Merin O, Rosenmann E, Dzigivker I, Bitran D, Yinnon AM, et al. Latent infective endocarditis: epidemiology and clinical characteristics of patients with unsuspected endocarditis detected after elective valve replacement. Department of Cardiothoracic Surgery, Shaare Zedek Medical Center, Jerusalem, Israel. Ann Thorac Surg. 2004;78: 1623-9.

5. Sexton DJ, Spelman D. Current best practices and guidelines. Assessment and management of complications in infective endocarditis. Cardiol Clin. 2003;21:273-82, vii-viii 